
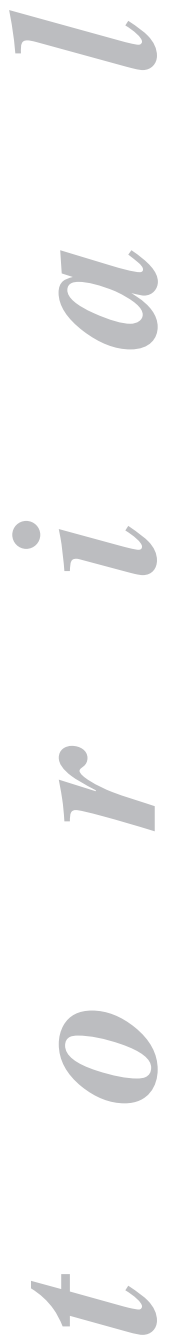

O
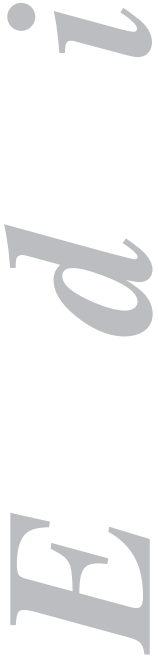

\section{Adalimumab en la enfermedad de Crohn: los datos de la vida real}

En este número de la Revista Española de Enfermedades Digestivas, el Grupo de Enfermedad Inflamatoria Intestinal del Hospital Clínico de San Carlos de Madrid, nos presenta los resultados obtenidos en su centro al tratar con adalimumab una serie de 22 pacientes con enfermedad de Crohn (EC) en los cuales la administración de infliximab había sido interrumpida, ya por efectos adversos graves, ya por pérdida de respuesta (1). Inician el tratamiento con una dosis estándar de $180 \mathrm{mg}$ y continúan con $80 \mathrm{mg}$ a las dos semanas y posteriormente $40 \mathrm{mg}$ cada 2 semanas. En algunos pacientes se acortaron los intervalos. Obtienen respuesta completa en 5 de 22 pacientes y respuesta parcial en otros 13. Analizan el mantenimiento de la respuesta en pacientes con enfermedad luminal, por un lado, y fistulosa por otro.

Se hace el seguimiento de 13 pacientes con enfermedad luminal (4 con respuesta completa y 9 con respuesta parcial). La respuesta se mantuvo al año en el 71,6\% y a los 24 meses en el $35,8 \%$, pero no todos los pacientes llegaron al final de este periodo. En cuanto a los datos en enfermedad fistulosa, no nos parece apropiado sacar conclusiones sobre el seguimiento del pequeño grupo de cinco pacientes con respuesta parcial o completa.

Emplearon pues el fármaco en una situación clínica absolutamente comparable con la que otros centros encuentran, y lo hicieron a las dosis que se recomiendan en la actualidad, durante un tiempo no establecido por los datos de la literatura, pero que sabemos que se puede prolongar al menos un año (2) o incluso dos, a tenor de los resultados presentados en el congreso de la European Crohn's and Colitis Organization de 2008 (3). Por ello, sus conclusiones nos interesan, ya que se van a referir a lo que nos encontramos diariamente en el tratamiento de personas con enfermedad inflamatoria intestinal (EII).

Quizás sea necesario explicar brevemente lo que es el adalimumab y cuáles son sus similitudes y diferencias con el único otro anticuerpo monoclonal anti-TNF $\alpha$ comercializado en España, el infliximab. Recordemos tan sólo, que se trata en ambos casos de moléculas de clase IgG1 que bloquean la acción del factor de necrosis tumoral alfa e inducen la apoptosis de clones linfocitarios activados, lo que resulta en una actividad antiinflamatoria potente y más o menos duradera (2).

La molécula que primero apareció en el panorama terapéutico de la enfermedad de Crohn, fue el infliximab. Sorprendió por su acción rápida y, en muchos casos, extremadamente brillante, en dos terrenos en los que pacientes y médicos nos habíamos visto hasta entonces muy atados de pies y manos: la enfermedad fistulosa de difícil manejo y la enfermedad luminal refractaria a esteroides. Sus problemas parecían estar relacionados con el coste, la dependencia del hospital (por su administración i.v.), la facilitación de infecciones o tumores, la capacidad inmunogénica (que daba lugar a reacciones infusionales y a fenómenos de tipo autoinmune) y la 
pérdida de eficacia, observada en forma de taquifilaxia, que sólo la prolongación de su uso ha venido a revelar. Parte de estos últimos problemas, pero no todos, se relacionan con el hecho de tratarse de una molécula quimérica, con una porción de origen murino.

El adalimumab, por otra parte, nació como respuesta a algunas de estas cuestiones. Se trata de una molécula humanizada, menos inmunogénica y de administración subcutánea. En teoría, no debería generar la producción de anticuerpos, aunque en la práctica estos se observan en una proporción de casos $(2,4)$. No parece que la asociación de inmunomoduladores tenga en su caso tanta importancia $(2,4,5)$. Carece, además, de reacciones infusionales (2). Pero, al igual que ocurre con el infliximab, es frecuente la pérdida de respuesta al fármaco, por lo que en el terreno práctico hemos observado que, con cierta frecuencia, es necesario escalar la dosis para mantener el efecto (6).

La experiencia con adalimumab es ya importante. El primer ensayo fundamental, denominado CLASSIC I, probó este fármaco en la inducción de respuesta y remisión en pacientes con enfermedad de Crohn (5). Concretamente, se trataba de un grupo de 299 pacientes, con EC moderada a grave, que no habían recibido previamente tratamiento anti-TNF $\alpha$. Se aleatorizaron los pacientes recibiendo diferentes dosis (semanas 0 y 2, adalimumab $40 \mathrm{mg} / 20 \mathrm{mg}, 80 \mathrm{mg} / 40 \mathrm{mg}$ o $160 \mathrm{mg} / 80 \mathrm{mg}$ ) de adalimumab s.c. y otros sólo placebo. El objetivo primario designado era la demostración de una diferencia significativa en las tasas de remisión $(\mathrm{CDAI}<150)$ en la semana 4 . Se demostró que la remisión se conseguía en el $12 \%$ del grupo placebo, frente al $18 \%$ en el grupo 40-20 ( $p=0,36), 24 \%$ en el grupo $80-40(p=0,06)$ y $36 \%$ en el grupo 160-80 ( $\mathrm{p}=0,001)$. No hubo diferencias en la tasa de efectos adversos y la conclusión del estudio fue que el adalimumab mejoró los resultados obtenidos en el grupo placebo en lo que se refiere a la inducción de la remisión de enfermedad de Crohn moderada o grave nunca tratada con anti-TNF $\alpha$. La dosis óptima, de hecho la única a la que esta diferencia era claramente significativa, fue la de $160 \mathrm{mg}$ al día 0 , seguida de $80 \mathrm{mg}$ a las 2 semanas.

Una experiencia española, coordinada por Joaquín Hinojosa, del Hospital de Sagunto, y bajo el impulso del Grupo Español de Trabajo en Enfermedad de Crohn y Colitis Ulcerosa (GETECCU), aportó datos adicionales directamente desde los primeros pacientes tratados en nuestro país por uso compasivo (7). Se recogieron datos de 50 pacientes, correspondientes a diferentes centros de la geografía española, con falta o pérdida de respuesta al infliximab. Los 50 pacientes fueron tratados con adalimumab en la pauta que, según el estudio CLASSIC I, se había mostrado más eficaz (160 mg s.c. en el día 0 , seguidos de $80 \mathrm{mg}$ en la semana 2). De los 36 pacientes con enfermedad de Crohn luminal se obtuvo respuesta (reducción de al menos 70 puntos en el CDAI) en el 83\% y remisión (CDAI < 150) en el 42\%. De los 22 con enfermedad fistulosa, cinco (23\%) consiguieron el cierre de las fístulas y en nueve (41\%) estas mejoraron (disminución al menos del $50 \%$ en el número de fístulas abiertas). No hubo efectos adversos graves por lo que los autores concluyeron que el adalimumab es adecuado en el tratamiento de la enfermedad de Crohn luminal o fistulosa con pérdida de respuesta o intolerancia al infliximab.

En el año 2007, nos llegaron los datos del estudio CLASSIC II (8). En este, se pretendía conocer los resultados del adalimumab administrado a largo plazo. Se incluyeron 276 pacientes del estudio CLASSIC I (es decir, nunca antes tratados con agentes biológicos). Primero, se administraron dos dosis de $40 \mathrm{mg}$ de adalimumab en las semanas 0 y 2. Luego se hicieron dos grupos de tratamiento. Por un lado, se procedió a aleatorizar a 55 que se encontraron en remisión en las semanas 0 y 4, a 
recibir mantenimiento con $40 \mathrm{mg}$ de adalimumab cada dos semanas, cada semana o con placebo, durante 56 semanas. Los pacientes de este grupo sin respuesta o con brote, recibieron adalimumab en abierto, $40 \mathrm{mg}$ cada dos semanas, seguido de adalimumab semanal si fuera necesario. El objetivo fue mantener la remisión (CDAI < 150 ) en los pacientes aleatorizados, hasta la semana 56. De estos 55 pacientes, el $79 \%$ de los tratados con adalimumab $40 \mathrm{mg}$ cada dos semanas y el $83 \%$ de los tratados con adalimumab semanal se mantenían en remisión en la semana 56, frente al $44 \%$ de los que recibieron placebo $(\mathrm{p}<0,05)$.

Por otro lado, los no respondedores a las semanas 0 y 4 recibieron adalimumab en abierto con $40 \mathrm{mg}$ cada dos semanas. Si no había respuesta o aparecía un brote, se incrementaba la dosis a $40 \mathrm{mg}$ semanales. De los 204 pacientes tratados en abierto, $93(46 \%)$ estaban en remisión a la semana 56. La tolerancia fue buena. La conclusión del estudio fue que se podía mantener la remisión con adalimumab a $40 \mathrm{mg}$ cada dos semanas.

En ese mismo año, el estudio CHARM (9) aportó más datos. Se administró el adalimumab en tratamiento abierto de inducción con $80 \mathrm{mg}$ en la semana 0 seguidos de $40 \mathrm{mg}$ en la semana 2. Luego, en la semana 4, se estratificó a los pacientes según su respuesta (disminución del CDAI $>70$ puntos) y se los aleatorizó a recibir tratamiento doble ciego con placebo, adalimumab $40 \mathrm{mg}$ cada 2 semanas o adalimumab $40 \mathrm{mg}$ semanales, hasta la semana 56 . El porcentaje de respondedores que mantuvieron la remisión fue mayor en los grupos de adalimumab cada 2 semanas o semanal frente a placebo, tanto en la semana $26(40,47$, y $17 \%$, respectivamente, p < $0,001)$ como en la semana $56(36,41$ y $12 \%$, respectivamente; $p<0,001)$. No hubo diferencias significativas en la eficacia entre ambas pautas de adalimumab. La conclusión fue que en los pacientes que responden al adalimumab, se puede mantener la respuesta con $40 \mathrm{mg}$ semanales o cada dos semanas.

Si bien no fue el objetivo primario del estudio, de los 64 pacientes con fístulas que drenaban activamente al inicio, fundamentalmente perianales, en la semana 56 estas habían cicatrizado completamente en el 33\% de los pacientes tratados con adalimumab (ambos grupos: $40 \mathrm{mg}$ cada 2 semanas y $40 \mathrm{mg}$ semanales) versus $13 \%$ en el grupo placebo.

Era muy importante conocer qué pasaba en los pacientes que ya habían sido tratados previamente con infliximab (aunque el estudio español proporcionaba ya algunos datos). En 2007, se conocieron los datos de un estudio denominado GAIN (6), en el que se incluyó a 301 pacientes que habían recibido infliximab pero que no lo habían tolerado o habían dejado de responder a él. A la entrada, la enfermedad de Crohn se encontraba activa, con un CDAI de 220-450. Se aleatorizó a los pacientes para recibir inducción con adalimumab 160-80 (semana 0 y 2) o placebo en los mismos momentos. El 21\% (34 de 159) de los pacientes tratados con adalimumab consiguieron la remisión en la semana 4 frente a 7\% (12 de 166) del grupo placebo ( $\mathrm{p}<0,001)$. Además de que hubo más remisiones, también hubo más respuestas. A la semana 4, disminuyó el CDAI 70 puntos o más en el 52\% de los pacientes tratados con adalimumab frente al $34 \%$ de los de los pacientes incluidos en el grupo placebo $(\mathrm{p}=0,001)$. No hubo efectos adversos graves, y la conclusión a la que se llegó fue que el adalimumab conseguía inducir la remisión con más frecuentemente que el placebo en pacientes con intolerancia o pérdida de respuesta a infliximab.

El adalimumab es pues un fármaco que ha demostrado su utilidad clínica y que tiene un perfil de seguridad más que aceptable. Entramos en la fase en la que nos irán llegando, por un lado, las experiencias que se puedan plantear en pacientes con otras enfermedades, concretamente, con colitis ulcerosa. Por otro lado, quizás más 
importante, iremos aprendiendo a perfeccionar el empleo de este fármaco, identificando los factores que predigan una mayor respuesta, colocándolo en una fase temporal adecuada dentro del tratamiento de la enfermedad de Crohn, y conociendo los problemas que su manejo a largo plazo pudieran plantear (10-13). Conoceremos sus fortalezas, entre las que cabe recordar sobre todo la comodidad de su administración, además de la ausencia de reacciones adversas. También aprenderemos sobre sus debilidades, entre las que tendremos que enfrentar la necesidad de escalar la dosis en el seguimiento (con el consiguiente aumento de costes) y los efectos adversos propios de los inmunomoduladores $(2,6,10)$.

López Palacios y cols. obtienen en su serie de pacientes con fracaso o intolerancia a infliximab una tasa apreciable de respuestas, empleando la pauta habitual y en porcentajes similares a los de experiencias previas parecidas (7). Pueden parecer cifras poco brillantes, pero hay que tener en cuenta que no se trata de pacientes vírgenes a los agentes biológicos y que ello habla de la complejidad de sus cuadros. Por otra parte, la medida de la respuesta mediante un índice clínico cualquiera, puede calificar a ciertas respuestas como parciales, cuando en realidad son satisfactorias para el paciente y, por eso, es adecuado analizar los pacientes que se beneficiaron en algún grado del tratamiento. La tasa más baja de respuesta en la enfermedad de Crohn fistulosa que los autores obtienen es, en nuestra opinión, reflejo del panorama más complicado de este subtipo de la enfermedad, en el que no todo está escrito, y que delinea una situación que requiere un enfoque multidisciplinar y paciente, $\mathrm{y}$ en la que probablemente aún haya que escribir nuevos capítulos.

En conclusión, el adalimumab es un nuevo fármaco que tiene propiedades interesantes y que sabemos que funciona y es seguro. Esperamos que en los próximos tiempos, las nuevas experiencias nacionales en las que el adalimumab se haya empleado, no en fracasos del infliximab, sino como primer tratamiento biológico, ofrezcan datos que lo sitúen verdaderamente dentro del panorama de la terapia de la enfermedad inflamatoria intestinal y ojalá nuestros pacientes encuentren en él una ayuda más, tan valiosa que les permita sobrellevar los problemas que provoca su enfermedad de Crohn.

\author{
A. López San Román, M. Van Domselaar y E. Garrido \\ Unidad de Enfermedad Inflamatoria Intestinal. Servicio de Gastroenterología. \\ Hospital Ramón y Cajal. Madrid
}

\title{
BIBLIOGRAFÍA
}

1. López Palacios N, Mendoza JL, Taxonera C, Lana R, Fuentes Ferrer M, Díaz-Rubio M. Tratamiento de inducción y mantenimiento con adalimumab en la enfermedad de Crohn: un estudio abierto. Rev Esp Enferm Dig 2008; 100: 676-681.

2. Panés J, Gomollón F, Taxonera C, Hinojosa J, Clofent J, Nos P. Crohn's disease: a review of current treatment with a focus on biologics. Drugs 2007; 67: 2511-37.

3. Panaccione R, Colombel JF, Sandborn WJ, Rutgeerts P, D'Haens GH, Lomax KG, et al. Adalimumab maintains long-term remission in Crohn's disease through 2 years. European Crohn's and Colitis Organization. Lyon, 2008.

4. Cottone M, Mocciaro F, Scimeca D. Adalimumab induction for Crohn's disease. Gastroenterology 2006; 130: 1929. 
5. Hanauer SB, Sandborn WJ, Rutgeerts P, Fedorak RN, Lukas M, MacIntosh D, et al. Human anti-tumor necrosis factor monoclonal antibody (adalimumab) in Crohn's disease: the CLASSIC-I trial. Gastroenterology 2006; 130: 323-33.

6. Sandborn WJ, Rutgeerts P, Enns R, Hanauer SB, Colombel JF, Panaccione R, et al. Adalimumab induction therapy for Crohn disease previously treated with infliximab: a randomized trial. Ann Intern Med 2007; 146: 829-38.

7. Hinojosa J, Gomollón F, García S, Bastida G, Cabriada JL, Saro C, et al on behalf of GETECCU. Efficacy and safety of short-term adalimumab treatment in patients with active Crohn's disease who lost response or showed intolerance to infliximab: a prospective, open-label, multicentre trial. Aliment Pharmacol Ther 2007; 25: 409-18.

8. Sandborn WJ, Hanauer SB, Rutgeerts P, Fedorak RN, Lukas M, MacIntosh DG, et al. Adalimumab for maintenance treatment of Crohn's disease: results of the CLASSIC II trial. Gut 2007; 56: 1232-9.

9. Colombel JF, Sandborn WJ, Rutgeerts P, Enns R, Hanauer SB, Panaccione R, et al. Adalimumab for maintenance of clinical response and remission in patients with Crohn's disease: the CHARM trial. Gastroenterology 2007; 132: 52-65.

10. Ricart E, García-Bosch O, Ordas I, Panes J. Are we giving biologics too late? The case for early versus late use. World J Gastroenterol 2008; 14: 5523-7.

11. Martín JM, Ricart JM, Alcácer J, Rausell N, Arana G. Adalimumab-induced lupus erythematosus. Lupus 2008; 17: 676-8.

12. Hinojosa J, Borrás-Blasco J, Maroto N, Rosique-Robles JD, Alos R, Casterá ME. Severe myalgia associated with adalimumab treatment in a patient with Crohn's disease. Ann Pharmacother 2008; 42: 1130-3.

13. Ramos-Casals M, Brito-Zerón P, Muñoz S, Soria N, Galiana D, Bertolaccini L, et al. Autoimmune diseases induced by TNF-targeted therapies: analysis of 233 cases. Medicine (Baltimore) 2007; 86: 242-51. 